\title{
Reprogramming plant specialized metabolism by manipulating protein kinases
}

\author{
Ruiqing Lyu ${ }^{1}$, Sanjay K. Singh ${ }^{1}$, Yongliang Liu ${ }^{1,2}$, Barunava Patra ${ }^{1}$, \\ Yan Zhou' ${ }^{1}$, Bingwu Wang ${ }^{3}$, Sitakanta Pattanaik ${ }^{1}$, Ling Yuan ${ }^{1,2 \bowtie}$ (D) \\ ${ }^{1}$ Department of Plant and Soil Sciences and the Kentucky Tobacco Research and Development Center, University of \\ Kentucky, 1401 University Drive, Lexington, KY 40546, USA \\ 2 South China Botanical Garden, Chinese Academy of Sciences, Guangzhou 510650, China \\ 3 Tobacco Breeding Center, Yunnan Academy of Tobacco Agricultural Sciences, Kunming 650021, Yunnan, China
}

Received: 5 March 2021 / Accepted: 5 June 2021 / Published online: 17 June 2021

\begin{abstract}
Being sessile, plants have evolved sophisticated mechanisms to balance between growth and defense to survive in the harsh environment. The transition from growth to defense is commonly achieved by factors, such as protein kinases (PKs) and transcription factors, that initiate signal transduction and regulate specialized metabolism. Plants produce an array of lineage-specific specialized metabolites for chemical defense and stress tolerance. Some of these molecules are also used by humans as drugs. However, many of these defense-responsive metabolites are toxic to plant cells and inhibitory to growth and development. Plants have, thus, evolved complex regulatory networks to balance the accumulation of the toxic metabolites. Perception of external stimuli is a vital part of the regulatory network. Protein kinase-mediated signaling activates a series of defense responses by phosphorylating the target proteins and translating the stimulus into downstream cellular signaling. As biosynthesis of specialized metabolites is triggered when plants perceive stimuli, a possible connection between PKs and specialized metabolism is well recognized. However, the roles of PKs in plant specialized metabolism have not received much attention until recently. Here, we summarize the recent advances in understanding PKs in plant specialized metabolism. We aim to highlight how the stimulatory signals are transduced, leading to the biosynthesis of corresponding metabolites. We discuss the post-translational regulation of specialized metabolism and provide insights into the mechanisms by which plants respond to the external signals. In addition, we propose possible strategies to increase the production of plant specialized metabolites in biotechnological applications using PKs.
\end{abstract}

Keywords Medicinal plants, Protein kinases, MAP kinase, Specialized metabolites, Post-translational regulation, Signal transduction

\section{INTRODUCTION}

Being sessile, plants cannot evade biotic and abiotic stresses. Plants possess sophisticated physical (structural) and chemical defense systems to combat pathogens and survive in the harsh environment.

$\bowtie$ Correspondence: lyuan3@uky.edu (L. Yuan)
Biosynthesis of specialized metabolites in response to various biotic or abiotic stresses is a key component of chemical defense. Unlike primary metabolites, specialized metabolites are not usually involved in the basal development of plants, and excessive accumulation of such metabolites often hinders or inhibits plant growth and development. Therefore, healthy plants usually accumulate very low levels of specialized metabolites; 
however, the biosynthesis is triggered rapidly when plants are subject to biotic or abiotic stress. Plants produce more than 200,000 specialized metabolites (Pott et al. 2019). Although the chemical nature of specialized metabolites varies in plants, the majority of them originate from the isoprenoid, phenylpropanoid, terpenoid, or fatty acid pathway (Dixon 2001). Many specialized metabolites are cytotoxic, capable of killing pathogenic microbes or insects, and are, thus, considered as chemical weapons of plants.

Biosynthesis of many (if not all) defense-associated specialized metabolites is coupled with the plant immune system that responds rapidly to external stimuli. The plant immune systems can be divided into two types: pathogen-associated molecular pattern (PAMP)-triggered immunity (PTI) and effector-triggered immunity (ETI) (Jones and Dangl 2006). PTI and ETI are accomplished by a series of protein kinases (PKs) for signal perception and transduction. PKs are a class of enzymes that use the phosphate group of ATP to phosphorylate target proteins (substrates). PKs are universal in eukaryotes. Genome sequence analysis of 25 major plant species identifies more than 26,000 PKs (Lehti-Shiu and Shiu 2012). The Arabidopsis genome contains more than 1000 genes encoding PKs (Wang et al. 2007). All PKs have a functional domain, consisting of 250-300 amino acids, for phosphate group transfer (Xu and Nussinov 1998). The functionally conserved PKs do not share high sequence identity outside of the functional domain (Xu and Nussinov 1998).

PKs play critical roles in the perception of external stimuli, especially in the plant immune system. The conserved motifs in PAMPs are recognized as ligands by relevant pattern recognition receptors (PRRs) in host plant cells (Mogensen 2009). Plant PRRs are receptor kinases and receptor-like kinases (RLKs) present on the plant cell surface and have been well reviewed (Couto and Zipfel 2016). In brief, RLKs have no kinase domain or intracellular signaling domain and functionally depend on receptor kinases. Both receptor kinases and RLKs contain a ligand-binding domain. Based on the protein structure of the ligand-binding domain, PRRs are classified into four groups (Couto and Zipfel 2016; Zipfel 2014): the leucine-rich repeat (LRR)-containing PRRs that bind the peptides released by microbial pathogens, the lysine motif (LysM) PRRs that recognize carbohydrate-based ligands, such as fungal chitin or bacterial peptidoglycan (Desaki et al. 2018), and lectintype PRRs with binding activity to extracellular ATP or bacterial lipopolysaccharides (LPS). As some pathogenic bacteria pierce plant cells to secrete virulence effector proteins directly into the plant cytoplasm, a class of intracellular nucleotide-binding domain leucine-rich repeat receptor kinases (NB-LRRs) specifically bind to the effector proteins (Noman et al. 2019; Wang and Chai 2020). The stimulus signal recognized by PRRs is then transferred to the receptor-like cytoplasmic kinases (RLCKs) by direct phosphorylation (Li et al. 2014a; Cui et al. 2018; Liang and Zhou 2018). A branched signaling cascade characterized by rapid ion-flux changes is primed within minutes, leading to the alteration of extracellular $\mathrm{pH}$ and reactive oxygen species (ROS) and elevation of cytosolic $\mathrm{Ca}^{2+}$ level. In turn, $\mathrm{Ca}^{2+}$-dependent protein kinase (CDPK) and mitogen-activated protein kinase (MAPK) signaling cascades drive the transcriptome and metabolome reprogramming, establishing defense response (Boller and Felix 2009; Seybold et al. 2014; Lee et al. 2015; Coppola et al. 2019).

In addition to their functions in plant immunity, PKs play vital roles in response to abiotic stress. Unlike transcription factors (TFs), the kinases are not considered as the executors that directly reshape the transcriptomes and metabolomes in plants as they lack DNA-binding domains for altering gene expression. PKs target multiple TFs which directly bind to the target gene promoters to regulate their activities. The important roles of TFs in regulating plant specialized metabolites have been intensively studied in model plants and medicinal plants (Patra et al. 2013). However, how kinases modulate these regulators, particularly in plant specialized metabolism under biotic and abiotic stresses, remains poorly understood. It is, thus, imperative to study how PKs regulate specialized metabolism through TFs.

Medicinal plants produce a vast array of specialized metabolites with antimicrobial and/or antioxidant activities. Plant specialized metabolites are the major determinants of the medical and commercial values of medicinal plants (Hussein and El-Anssary 2019; Anand et al. 2019). Some specialized metabolites are used as drugs. More than $25 \%$ of clinical anthropic drugs are derived from medicinal plants (Wurtzel and Kutchan 2016). Well-known examples include the anti-malarial drug artemisinin from Artemisia annua (Weathers et al. 2006), as well as the anticancer drugs Taxol from Taxus spp. (Lenka et al. 2015) and vincristine and vinblastine from Catharanthus roseus (Dugé de Bernonville et al. 2017). However, while toxic to pests or microbes and inhibitory to animal cells, many specialized metabolites are also cytotoxic to plants (Isah 2019). Therefore, excessive synthesis of these metabolites may inhibit the growth and development of plants. In addition, specialized metabolism is energy-intensive, competing with resources for plant growth. The coupling of specialized metabolite biosynthesis and the immune system makes it possible for the plants to instantaneously synthesize 
the toxic metabolites after sensing biotic stress without overly affecting growth and development (Gorlenko et al. 2020). Additionally, transcriptomic analysis of the medicinal plants, such as Echinacea purpurea (Tahmasebi et al. 2019), C. roseus (Verma et al. 2014; Pan et al. 2018), Salvia miltiorrhiza (Wenping et al. 2011), and Valeriana officinalis (Yeo et al. 2013), reveals that many protein kinase-encoding genes are differentially expressed in response to external stimuli.

Only a limited number of studies so far demonstrate the possible roles of PKs in regulating plant specialized metabolism. Even less known is the regulatory mechanisms that govern such processes. We, thus, compel to review and discuss the recent studies on (i) the possible relationship between PKs in the plant immune system and specialized metabolite biosynthesis, (ii) whether the production of different specialized metabolites in the same plant species requires the activation of different kinase signaling pathways, and (iii) whether the kinase signaling pathways are conserved across plants, especially medicinal plants. Additionally, we intend to provide convincing supports for using PKs to systematically modify the plant metabolic systems to increase the production of medicinally valuable specialized metabolites.

\section{Stress-induced specialized metabolism in plants}

Research into secondary metabolism dates back to 1910, when Albrecht Kossel, a Nobel laureate in chemistry, first created the term "secondary metabolites" to distinguish them from primary metabolites (Jones 1953; Hartmann 2008). Not until 1970s, the important functions of secondary metabolites in plants have been realized. "Secondary metabolites" are classified as molecules with no function in plant growth and development. These metabolites are usually synthesized at very low levels (less than $1 \%$ of total carbon) (Bourgaud et al. 2001) and rapidly induced when the environment is unfavorable (Ebel 1979). For example, leaf resins of Larrea spp. over-accumulate phenylpropanoid derivatives when exposing to ultraviolet (UV) radiation (Rhoades 1977). The abiotic stresses, such as drought, high salinity, excess light, and cold, trigger the production of anthocyanins in Arabidopsis thaliana (Christie et al. 1994; Cominelli et al. 2008; Nakabayashi et al. 2014; Isah 2019). Anthocyanin induction is more obvious during the biotic stresses by pathogenic microbes, pests, and herbivores (Bennett and Wallsgrove 1994; Ramakrishna and Ravishankar 2011; Ashraf et al. 2018). Upon pathogen infection, $A$. thaliana also produces other secondary metabolites, including indole and indole-sulfur compounds, glucosinolates, benzenoids, terpenes, and fatty acid derivatives (reviewed in D'Auria and Gershenzon 2005). These metabolites induced by biotic or abiotic stress act as antimicrobial molecules or antioxidants. As more and more secondary metabolites are identified in plants, their important roles in plant growth and defense have also been gradually recognized. Some secondary metabolites are also produced by other organisms including bacteria and fungi (Turner 1971; Pietra 1997), the roles of these metabolites are far from secondary. The term "specialized metabolites" has, thus, emerged to replace secondary metabolites (Pichersky and Lewinsohn 2011; Dixon 2001; Kutchan 2001).

In plants, specialized metabolites are classified into terpenes, flavonoids, N-containing compounds (alkaloids), and phenylpropanoids according to their chemical structures (Wang et al. 2019). They are synthesized from the end products or intermediates of the TCA cycle, photosynthesis, and other processes. The biosynthesis of many plant specialized metabolites is induced by various biotic or abiotic stresses. The transcriptional regulation of biosynthesis of specialized metabolites in plants has been intensively investigated (Shoji and Yuan 2021; Cao et al. 2020; Meraj et al. 2020; Vom Endt et al. 2002; Yang et al. 2012; Patra et al. 2013). The majority of the TFs involved in the biosynthesis of specialized metabolites are from the families of R2R3 MYB, bHLH (basic helix-loop-helix), AP2/ERF (APETALA2/ethylene-responsive factor), WRKY, Zinc finger, and NAC factor (Cao et al. 2020; Shoji and Yuan 2021; Singh et al. 2021; Meraj et al. 2020; Yang et al. 2012). For example, during salt stress, MYB111 binds to the cis-elements in the promoter of chalcone synthase $(\mathrm{CHS})$, flavanone carboxylase $(\mathrm{F} 3 \mathrm{H})$, flavanol synthase 1 (FLS1), upregulates their transcripts which ultimately trigger the accumulation of the flavonoids ( $\mathrm{Li}$ et al. 2019). Flavonoids act as antioxidants in plants (Pietta 2000). The absence of MYB111 in the myb111 mutant dramatically induces the ROS level upon salt treatment (Li et al. 2019). A recent study found that BHLH IRIDOID SYNTHESIS 3 (BIS3), a member of the bHLH TF family, transactivates iridoid pathway genes and positively regulates terpenoid indole alkaloid (TIA) biosynthesis in C. roseus upon the induction of jasmonic acid (JA) (Singh et al. 2021).

\section{Plant PKs and specialized metabolites}

In addition to transcriptional regulation, post-translational regulation, such as phosphorylation, is evident in many metabolic and developmental pathways. Phosphorylation activates a series of signaling cascades that regulate plant responses to biotic and abiotic stresses. 
An increasing number of studies have shown that many TFs are the substrates of PKs. Phosphorylation can regulate the activity, interactions, and subcellular localization of target proteins (Lee and Yaffe 2016). Based on subcellular localization, PKs can be divided into two broad categories: the ones associated with cell wall or plasma membrane via transmembrane domain(s), and those that resided in the cytosol, nucleus, or connect with organelles. Most of the available studies focus on the roles of PRRs, cell wall-associated kinases (WAKs), and mitogen-activated protein kinases (MAPKs) since they are critical in signaling perception and transduction (Erb and Reymond 2019). MAPKs can target the TF regulators and TFs to regulate the expression of defense-associated genes (Ng et al. 2018). MAPKs are also one of the most studied kinases in medicinal plants (Asai et al. 2002; Xu et al. 2008; Taj et al. 2010; Raina et al. 2012; Paul et al. 2017). Hence, we focus on the PRRs, WAKs, and MAPKs (Fig. 1), and highlight some kinases with direct roles in specialized metabolism (Table 1).

\section{Cell wall-associated kinases}

WAKs are related to cell wall-associated defense response, such as strengthening the cell integrity by triggering the biosynthesis of cellulose and phytoalexin (Hu et al. 2017). The Arabidopsis WAK family has five members. In monocot, e.g., rice, the WAK family has more than 100 members. Recent studies have reported the roles of WAKs in fungal resistance and the primary or specialized metabolism in model species (Diener and Ausubel 2005; Harkenrider et al. 2016; Zhang et al. 2017). The newly identified ZmWAK-RLK1 in maize

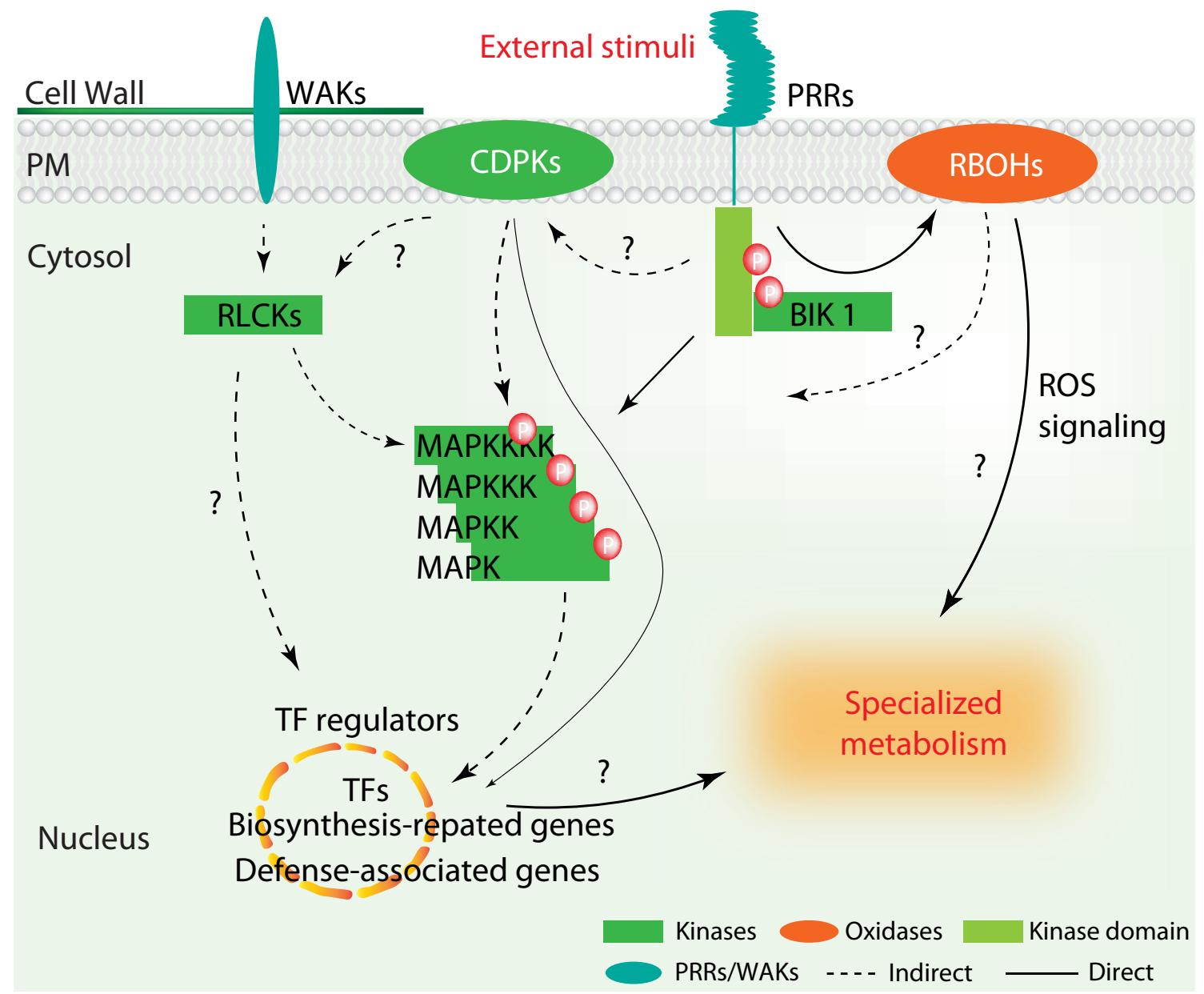

Fig. 1 A potential role of PKs in regulating specialized metabolism in medicinal plants. External stimuli are sensed by membrane-located kinases, such as wall-associated kinases (WAKs) and pathogen-associated molecular patterns (PAMPs) recognition receptor-like kinases (PRRs). The perception activates downstream kinases including cytosolic receptor-like kinases (RLCKs) and MAP kinase cascade, which eventually triggers the transcriptomic and metabolic reprogramming. PRRs may also connect with NADPH oxidases (RBOHs) or Ca ${ }^{2+}$. dependent kinases (CDPKs) to induce ROS $/ \mathrm{Ca}^{2+}$-dependent signaling cascades, that may contribute to the regulation of specialized metabolism. TFs transcription factors; PM plasma membrane 
Table 1 Kinases involved in specialized metabolism

\begin{tabular}{|c|c|c|c|c|}
\hline Kinase & $\begin{array}{l}\text { Kinase } \\
\text { family }\end{array}$ & Plant species & Specialized metabolite & References \\
\hline MKK9 & MAPKs & $\begin{array}{l}\text { Arabidopsis } \\
\text { thaliana }\end{array}$ & Camalexin & Xu et al. (2008) \\
\hline MPK3, МРК6 & MAPKs & A. thaliana & Camalexin & Mao et al. (2011) \\
\hline МАРККК $\alpha$ & MAPKs & A. thaliana & Camalexin & Ren et al. (2008) \\
\hline MEKK1 & MAPKs & A. thaliana & Camalexin & Ren et al. (2008) \\
\hline MPK3, MPK6 & MAPKs & A. thaliana & Indole glucosinolates & Xu et al. (2016) \\
\hline SmMAPK3 & MAPKs & Salvia miltiorrhiza & Tanshinone & Xie et al. (2020) \\
\hline SmSnRK2.6 & SnRKs & S. miltiorrhiza & Phenolic acid & Jia et al. (2017) \\
\hline CrMPK3 & MAPKs & $\begin{array}{l}\text { Catharanthus } \\
\text { roseus }\end{array}$ & Monoterpenoid indole alkaloids & Raina et al. (2012) \\
\hline CrMPKK1 & MAPKs & C. roseus & Monoterpenoid indole alkaloids & Paul et al. (2017) \\
\hline MdSOS2L1 & CIPKs & Apple & $\begin{array}{l}\text { Procyanidin biosynthesis and malate } \\
\text { metabolism }\end{array}$ & Hu et al. (2016b) \\
\hline MdHXK1 & Hexokinase & Apple & Anthocyanins & Hu et al. (2016a) \\
\hline $\begin{array}{l}\text { OsMPK3, OsMPK4, } \\
\text { OsMPK6 }\end{array}$ & MAPKs & Rice & Phytoalexin & $\begin{array}{l}\text { Kishi-Kaboshi et al. } \\
\text { (2010) }\end{array}$ \\
\hline$M h S n R K 1$ & SnRKs & Malus hupehensis & Ripening-related metabolism & Wang et al. (2012) \\
\hline PpSnRK1a & SnRKs & Peach & Ripening-related metabolism & \\
\hline AaSnRk2.6 & SnRKs & Artemisia annua & Artemisinin & Zhang et al. (2015) \\
\hline AKIN10 & SnRKs & A. thaliana etc & Terpenes & Robertlee et al. (2017) \\
\hline MAPKK1 (NtJAM1) & MAPKs & Tobacco & Nicotine & De Boer et al. (2011) \\
\hline NtMPK4 & MAPKs & Tobacco & Nicotine & Liu et al. (2021) \\
\hline
\end{tabular}

affects fungal resistance by altering the level of benzoxazinoids (BXs) (Yang et al. 2019). BXs are derived from indole-3-glycerophosphate (IGP) in the shikimate pathway. Mutation of ZmWAK-RLK1 leads to a decrease of BXs in maize compared to that in the wild-type plants after fungal infection. However, it is not known how ZmWAK-RLK1 regulates BX biosynthesis. As the perception of stimuli is conserved in the higher plants, WAKs may have a similar function in medicinal plants.

So far, no study of WAKs in medicinal plants has been reported, but transcriptomic analysis has identified the possible involvement of WAKs in response to stress and metabolism. Rheum australe is an endangered medicinal plant that produces anthraquinones and stilbenoids. $R$. australe grown at high altitudes in the Himalayas exposes to low temperature and high UV radiation. Anthraquinones are the precursors of anticancer drugs and have antioxidant activity (Campos-Martin et al. 2006). Stilbenoids act as antioxidants that can undergo intramolecular cyclization under UV radiation (Chong et al. 2009). Transcriptomic analysis reveals that the MAPK cascades, CDPKs, and WAKs are upregulated in the plants grown in natural conditions compared to those in growth chambers (Mala et al. 2021).

\section{Plasma membrane-located PKs}

The majority of PKs located in the cell wall, plasma membrane, or extracellular parts are PRRs. An Arabidopsis PRR, FLAGELLIN-SENSITIVE 2 (FLS2), activates BOTRYTIS-INDUCED KINASE 1 (BIK1) upon pathogen infection (Lu et al. 2010). BIK1 is a receptor-like cytoplasmic kinase (RLCK) that directly phosphorylates the respiratory burst oxidase homolog protein $\mathrm{D}$ (RBOHD). RBOHD is an NADPH oxidase involved in triggering the accumulation of ROS (Kadota et al. 2014; Li et al. 2014a). RBOHD and its homologs produce ROS through an NADP-malic enzyme (ME)-catalyzed reaction. ME coverts L-malate to pyruvate and produce carbon dioxide and NADPH (Detarsio et al. 2003). Pyruvate and glyceraldehyde-3-phosphate are the starting molecules of the methylerythritol-4-phosphate (MEP) pathway (Rodríguez-Concepción and Boronat 2002). The mevalonate (MVA) and MEP pathways produce all plant terpenoids, the largest family of specialized metabolites (Yazaki et al. 2017). RBOHs accept the electrons from NADPH and generate superoxide. Superoxide can spontaneously form hydrogen peroxide in plants. Both superoxide and hydrogen peroxide are ROS, acting as toxic molecules as well as secondary massagers. The 
plant cells synthesize antioxidants, some of which are specialized metabolites, such as flavonoids, phenolic acid, and carotenoids, to scavenge over-accumulated ROS (Chapman et al. 2019). Meanwhile, ROS signaling may crosstalk with kinase signaling and hormone signaling to regulate defense response (Waszczak et al. 2018).

\section{Sucrose nonfermenting-1 kinases}

Sucrose nonfermenting-1 kinases (SnRKs) are first identified in yeast (Saccharomyces cerevisiae). SnRKs can be divided into SnRK1, SnRK2, and SnRK3 subfamilies; members of the three subfamilies are involved in nitrogen, sucrose, and lipid metabolism (reviewed in Halford and Hey 2009). Overexpression of MhSnRK1 in tomato (Solanum lycopersicum L.) triggers fruit ripening (Wang et al. 2012). Overexpression of PpSnRK1 $\alpha$, a homolog of MhSnRK1 in peach (Prunus persica (L.)), also induces early fruit ripening in tomato (Yu et al. 2018). Yeast two-hybrid assay shows that SnRK1 interacts with the TF SIRIN (Yu et al. 2018), a MADS-box TF that regulates fruit ripening (Vrebalov et al. 2002). Ripening is a vital developmental process that results in a series of metabolic changes in fruits. Plants synthesize alkaloids and tannins during the early phase of the ripening process. These metabolites act as antifeedants which make the ripen fruits less favorable for animals (Prasanna et al. 2007). These antifeedant molecules are then replaced by sucrose and other specialized metabolites, such as lycopene, when fruits ripened.

SnRKs also regulate the biosynthesis of other specialized metabolites. The SnRK2.6, a member of the SnRK2 subfamily, regulates the levels of phenolic acids and tanshinoes in Salvia miltiorrhiza (Wang and Wang 2011). SnRK2.6 interacts with and activates the ABAresponsive $\mathrm{TF}$ SmAREB1 (ABA-responsive element ABRE-binding protein 1). Transgenic plants overexpressing SmSnRK2.6 have similar phenolic acid levels as the plants overexpressing SmAREB1 (Wang and Wang 2011). SnRKs also control terpene biosynthesis by regulating the activity of 3-hydroxy-3-methylglutaryl Coenzyme A Reductase (HMGR), the key rate-limiting enzyme in the mevalonic acid (MVA) pathway (Li et al. 2014b). The Arabidopsis SnRK1 AKIN10 negatively regulates the activity of HMGR by phosphorylation (Robertlee et al. 2017).

\section{Mitogen-activated protein kinases}

The MAPK cascade is an ancient signaling pathway prevalent in eukaryotes. The protein components in the cascade can be classified into four groups based on their positions in the pathway from downstream to upstream: MAP kinases (MAPKs), MAPK kinases (MAPKKs), MAPKK kinases (MAPKKKs), and MAPKKK kinases (MAPKKKKs). The upstream MAP kinases (such as MAPKKKs) can sequentially activate downstream kinases (MAPKKs), but not vice versa. Some MAPKKs have a conserved MAPK-docking motif K/R-K/R-K/R-X1-6-L-X$\mathrm{L} / \mathrm{V} / \mathrm{I}$ at the $\mathrm{N}$-termini but no transmembrane domain (Jiang and Chu 2018). Some MAPKs have a common C-terminal docking domain (CD) ([LH][LHY]Dxx[$\mathrm{DE}] \mathrm{xx}[\mathrm{DE}] \mathrm{EPxC}$; $\mathrm{x}$ represents any amino acid) that functions as a docking site for MAPKKs, phosphatases, and other protein substrates (Group et al. 2002). MAPKs function as signaling intermediates, which need to be triggered by the upstream receptor signals and perceived by downstream response components. The upstream receptor signals are derived from a series of membrane-associated kinases, such as PRRs. The downstream response components include enzymes, TFs, and PKs (Nakagami et al. 2005; Taj et al. 2010; Bigeard and Hirt 2018). MAPK signaling is involved in many biotic and abiotic stress responses and has been well reviewed (Taj et al. 2010; Nakagami et al. 2005; He et al. 2020). Accumulating evidence also suggests the vital roles of MAPKs in the biosynthesis of specialized metabolites. Here we discuss the post-translational regulation of important plant specialized metabolites by MAPKs.

\section{Anthocyanins}

Flavonoids are one of the most extensively studied plant specialized metabolites with antimicrobial and antioxidants activities. Anthocyanins are a family of flavonoids accumulated in flowers, fruits, and vegetables. Anthocyanin biosynthesis is mainly controlled by three families of TFs, the R2R3 MYBs, bHLH, and WD-repeat proteins that form the MYB-bHLH-WD40 (MBW) complex. In Arabidopsis, the R2R3 MYB TFs MYB75 (PAP1), MYB90 (PAP2), MYB113, and MYB114 (Gonzalez et al. 2008; Borevitz et al. 2000), the bHLH TFs TT8, GLABRA 3 (GL3), and ENHANCER OF GLABRA 3 (EGL3), and the WD40 TF TTG1 are positive regulators of anthocyanin (Gonzalez et al. 2008; Patra et al. 2013). Recent studies using Arabidopsis elucidate both positive and negative regulatory roles of MAPKs in anthocyanin biosynthesis. Light-induced anthocyanin biosynthesis is positively regulated by MPK4. Light rapidly activates MPK4, which in turn interacts with and phosphorylates MYB75 to enhance anthocyanin accumulation in Arabidopsis. Phosphorylation of MYB75 by MPK4 increases its stability but not alters its binding to the target gene promoters (Li et al. 2016). The Arabidopsis MAPKK6 is a 
negative regulator of anthocyanin biosynthesis. The mapkk6 mutant accumulates anthocyanin in leaves, and the expression of MYB75 is higher in the mutant compared to wild type (Wersch et al. 2018).

\section{Camalexin}

Camalexin is an indole alkaloid phytoalexin, acting as an antimicrobial molecule in Brassicaceae species (Glawischnig 2007). Camalexin biosynthesis is regulated by the WRKY TF WRKY33 and the R2R3 MYBs, MYB34, MYB51, and MYB122 (Birkenbihl et al. 2012; Frerigmann et al. 2015). WRKY33 directly binds to the promoter of Phytoalexin deficient 3 (PAD3), a camalexin biosynthetic gene (Zhou et al. 1999). WRKY33 is the target of MPK3/MPK6 (Mao et al. 2011). The upstream of MPK3/MPK6 is MAPKK (MAPKK $\alpha$ ) and MAPKKK(MEKK1), as the expression of the constitutively active form of MAPKK or MAPKKK promotes the camalexin biosynthesis (Ren et al. 2008). In addition, the pathogen-responsive Calcium-dependent protein kinase5 (CPK5) and CPK6 in Arabidopsis also regulate the camalexin biosynthesis by phosphorylating WRKY33 (Zhou et al. 2020). Phosphorylation of WRKY33 by MPK3/6 increases its transactivation, whereas CPK5/ CPK6 enhances DNA binding of WRKY33 (Zhou et al. 2020), suggesting that CPKs and MAPKs may function cooperatively (Zhou et al. 2020; Yang et al. 2020). The orthologs of MPK3/MPK4/MPK6 in rice also function in regulating the phytoalexin biosynthesis (Kishi-Kaboshi et al. 2010). In addition, MPKK9 and MPK4 are also involved in the regulation of camalexin biosynthesis in Arabidopsis. Overexpression of the wild-type or the phospho-mimic MAPKK9 induces camalexin pathway gene expression and camalexin accumulation in Arabidopsis. This MPKK9-induced camalexin biosynthesis requires the activation of the two downstream kinases, MAPK3/MAPK6 (Xu et al. 2008). Another study shows that MPK4 exists as a nuclear complex with WRKY33 and MAP Kinase Substrate 1 (MKS1) in the absence of pathogens. Upon challenged with pathogens, MPK4 is activated and phosphorylates MKS1, leading to the release of WRKY33 from the complex, which in turn activates camalexin biosynthesis (Qiu et al. 2008).

\section{Monoterpenoid indole alkaloids}

C. roseus produces over 130 monoterpenoid indole alkaloids (MIAs), of which vinblastine and vincristine are important chemotherapeutic agents (Neuss and Neuss 1990; Qu et al. 2019). MIA production is triggered by pathogen infection, wounding, UV-B, and methyl jasmonate (MeJA), the methyl ester of the defense hormone jasmonic acid (JA) (Pan et al. 2018; Gomi 2020). MIA biosynthesis is transcriptionally regulated by a network of TFs, including the AP2/ERFs ORCAs (ORCA2/ORCA3/ORCA4/ ORCA5/ORCA6), bHLH TFs (CrMYC2, BIS1/BIS2/BIS3, and RMT1), the WRKY TF (CrWRKY1), GATA-family TF (CrGATA1), and bZIP factors (GBF1 and GBF2) (Liu et al. 2019; Menke et al. 1999; Paul et al. 2017, 2020; Van Der Fits and Memelink 2000; Van Moerkercke et al. 2015, 2016; Zhang et al. 2011; Patra et al. 2018; Li et al. 2013; Suttipanta et al. 2011; Singh et al. 2020, 2021). The ORCAs, BISs, CrMYC2, and CrGATA1 are positive regulators, whereas RMT and GBFs are negative of MIA biosynthesis. Phosphorylation plays a role in MIA accumulation in Catharanthus. MeJA induces the accumulation of MIAs in $C$. roseus by activating the MAPK signaling cascade. Transient overexpression of CrMPK3 in C. roseus leaves upregulates the transcripts of genes associated with MIA biosynthesis and increases the production of MIAs, including serpentine, vincristine, vindoline, and catharanthine (Raina et al. 2012). CrMPK3 and its close homolog CrMPK6 are substrates of CrMAPKK1, the homolog of Arabidopsis MAPKK9 (Paul et al. 2017). CrMAPKK1 significantly enhances the transactivation activity of CrMYC2, ORCA3, ORCA4, or ORCA5 on the MIA pathway gene promoters in plant cells. In addition, overexpression of CrMAPKK1significantly induces MIA pathway gene expression and increases MIA accumulation in C. roseus hairy roots (Paul et al. 2017).

\section{Indole glucosinolates}

Glucosinolates are a group of thioglucosides found in the Brassicaceae family. The group includes the tryptophan-derived indole glucosinolates (IGS) and methionine-derived aliphatic glucosinolates (AGS). Glucosinolates and the derivatives serve as defense molecules against insect herbivores and pathogens. R2R3 MYBs (MYB34, MYB51, and MYB122), bHLH TFs (MYC2, MYC3, and MYC4), and ERF6 regulate IGS biosynthesis in Arabidopsis. The MAP kinases MPK3/ MPK6 are involved in IGS biosynthesis in response to pathogen infection. ERF6 is directly phosphorylated by MPK3/MPK6 to activate IGS biosynthesis. In addition, MYB51 and MYB122 are also involved in the MAPK signaling pathway (Xu et al. 2016; Yang et al. 2020).

\section{Nicotine}

Nicotine is the major alkaloid accumulated in tobacco. Similar to the MIA regulation in Catharanthus, the JAresponsive ERF189, ERF199, ERF221, and NtMYC2 are the major regulators of nicotine biosynthesis in tobacco. 
MAPK signaling is also involved in nicotine biosynthesis. Transient overexpression of the JA-factor stimulating MAPKK1 (NtJAM1) with the AP2/ERF ORC1 (ERF221) significantly activates the nicotine pathway gene promoters in tobacco cells (De Boer et al. 2011). NtORC1 is a homolog of CrORCA3. In Arabidopsis, MPK4 regulates light-induced biosynthesis of anthocyanin ( $\mathrm{Li}$ et al. 2016) and pathogen-induced camalexin (Qiu et al. 2008). A recent study shows that NtMPK4, a homolog of AtMPK4, regulates nicotine biosynthesis by activating ERF221. Conditional overexpression of NtMPK4 in hairy roots upregulates nicotine pathway gene expression and increases nicotine accumulation. In addition, co-expression of NtMPK4 with ERF221 significantly activates the nicotine pathway gene promoter compared to expressing ERF221 alone (Liu et al. 2021).

\section{Tanshinone}

The functions of MAPKs seem to be conserved in medicinal plants that produce unique specialized metabolites. In Salvia miltiorrhiza, MAPK seems to be associated with the biosynthesis of tanshinone (Xie et al. 2020). S. miltiorrhiza is a traditional herb in Chinese medicine. The roots of $S$. miltiorrhiza are rich in salvianolic acid and tanshinone. Tanshinone has significant medicinal potentials and is shown to inhibit the infection and replication of the viruses SARS-CoV-3CL ${ }^{\text {pro }}$ and $\mathrm{CL}^{\text {pro }}$ (Park et al. 2012). Genome-wide analysis has revealed $S$. miltiorrhiza genome harbors18 MAPKs, 9 MAPKKs, 83 MAPKKKs, and 6 MAPKKKKs. Yeast twohybrid assay shows that SmMAPK3 interacts with SmMYB36, a TF that promotes the biosynthesis of tanshinone in the hairy roots of $S$. miltiorrhiza (Ding et al. 2017).

\section{Artemisinin}

A. annua is a traditional Chinese herb that produces the sesquiterpene lactone artemisinin with anti-malarial activity. Artemisinin biosynthesis is positively regulated by the abscisic acid (ABA)-responsive basic leucine zipper TF AabZIP1, which binds to the promoter of genes involved in artemisinin biosynthesis (Zhang et al. 2015). The ABA-responsive Artemisinin biosynthesis promoting kinase 1 (AaAPK1), a SnRK2 in A. annua, interacts with and phosphorylates AabZIP1 to regulate the biosynthesis of artemisinin (Zhang et al. 2018).

\section{Reprogramming plant specialized metabolism by manipulating PKs}

The studies on different metabolic pathways collectively suggest that protein phosphorylation occurs in many specialized metabolic pathways. PKs are vital parts of the gene regulatory networks that control the biosynthesis of plant specialized metabolites in response to various biotic and abiotic factors. In addition, as defense response and specialized metabolite biosynthesis are well connected, many PKs involved in plant defense response are potential regulators of specialized metabolism. Furthermore, homologous kinases regulate structurally diverse specialized metabolites in different plant species. PKs, thus, can serve as potential tools to reprogram metabolic pathways in plants. Constitutively, active kinases have been used to activate metabolic pathways to overproduce certain specialized metabolites in plants. For example, the transgenic Arabidopsis lines expressing the constitutively active forms of MAPKKK $\alpha$ and MEKK1 ( $\triangle$ MAPKKK $\alpha$ and $\Delta$ MEKK1), driven by the DEX-inducible promoter, rapidly accumulates camalexin after the induction by DEX (Ren et al. 2008). Identification of candidate kinases, modification of the phosphorylation sites, and generation of transgenic plants overexpressing active kinase(s) are attractive approaches to reprogram metabolic pathways in plants. By secondary mutagenesis and genetic screening, plants that are tolerant to toxic metabolites with little growth inhibition can be identified (Fig. 2).

The modulation of metabolites can alternatively be achieved by overexpressing the native versions of kinases. Overexpression of CrMAPKK1 elevates the levels of MIAs in C. roseus (Paul et al. 2017). Overexpression of apple Hexokinase 1 (MdHXK1) triggers the accumulation of anthocyanin biosynthesis in apple calli and fruits (Hu et al. 2016a). The level of anthocyanin induced by overexpressing MdHXK1 is much higher than that of calli overexpressing the TF MdbHLH3 $\mathrm{CHu}$ et al. 2016a). The MdHXK1-mediated phosphorylation of MdbHLH3 occurs at the $\operatorname{Ser}^{361}$ site enhances the transcriptional activity of MdbHLH3. Loss of phosphorylation by substituting the amino acid $\mathrm{Ser}^{361}$ to $\mathrm{Ala}^{361}$ in MdbHLH3 lowers the anthocyanin production in transgenic calli ( $\mathrm{Hu}$ et al. 2016a). As activation of MdHXK1 requires glucose, whereas a constitutively active form of MdHXK1 may promote the production of anthocyanins without glucose treatment. The hexokinases are conserved across the plant kingdom, overexpression of HXK1 may also reshape the metabolism in other species. However, high hexokinase activity results in reduced fruit and seed size (Menu et al. 2004), and overexpression hexokinase has inhibitory effects on 


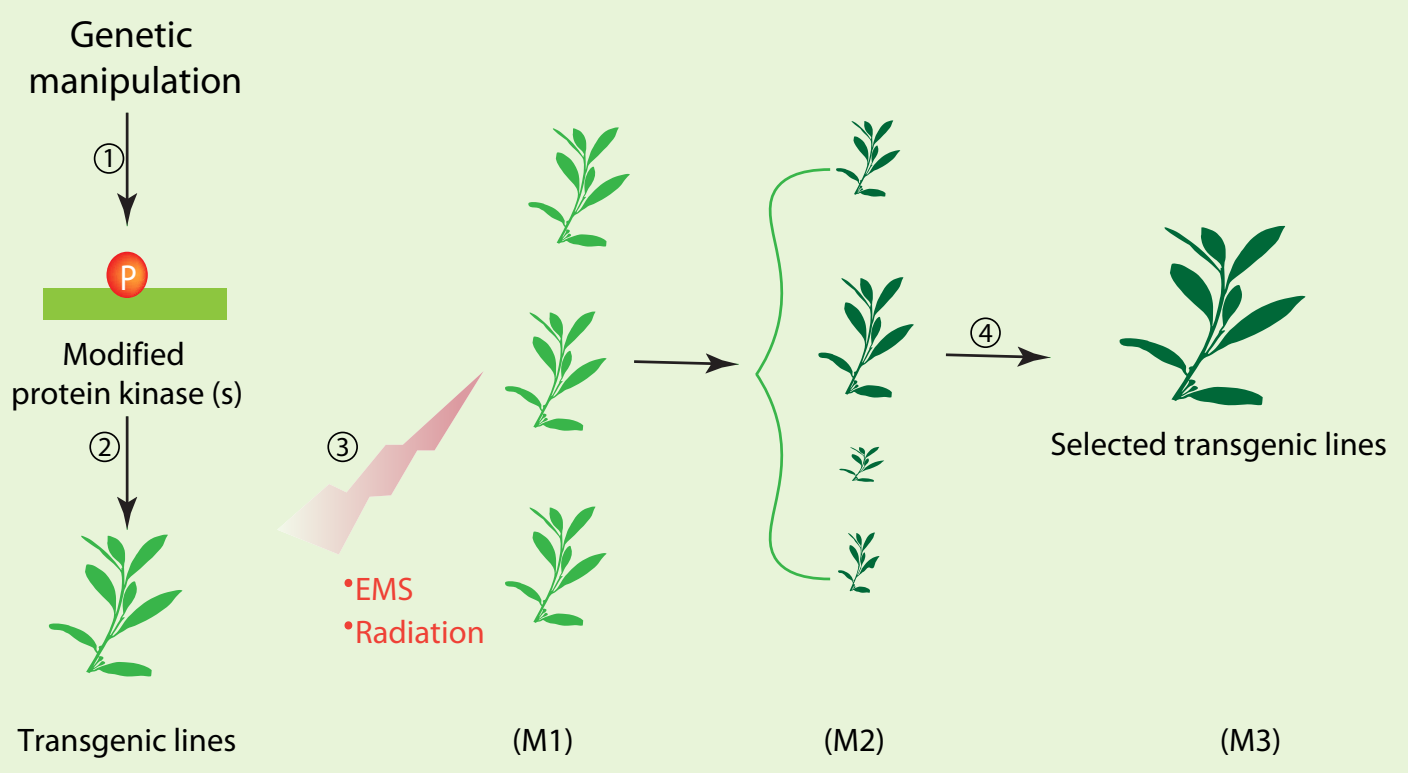

Fig. 2 A strategy to screen for a transgenic plan that resists increased toxic metabolites. Modification of a kinase (1) in transgenic plants (2) by overexpressing or gene-editing (knock-in mutagenesis) an engineered kinase that is either constitutively activated (phosphomimic) or with enhanced stability. " $\mathrm{P}$ " represents the phosphorylation of a protein kinase resulting in modified activity. The transgenic plants will be screened for increased metabolite accumulation and normal growth. In case the increase of metabolite accumulation causes growth inhibition, a secondary mutagenesis (3) using chemical means, such as ethyl methanesulfonate (EMS) or irradiation, will be employed to generate a mutant population (M1) with various growth phenotypes (plants with varying sizes in M2 population), from which a line (M3) with high metabolite accumulation and normal growth will be selected for futher breeding

plant development (Dai et al. 1999; Kelly et al. 2012), suggesting the necessity of secondary mutagenesis to screen for a transgenic plant without growth defects or achieving conditional expression using inducible system (Borghi 2010).

Kinases may also negatively regulate specialized metabolism. Overexpression of the activated version of MKK9 in Arabidopsis results in less anthocyanin under low nitrogen condition compared to the wild-type and the transgenic line expressing the native version of MKK9 (Luo et al. 2017). When a toxic or undesirable metabolite needs to be reduced, ectopic expression of a kinase with negative regulatory activity, or knockout of a kinase with positive activity using genome editing technology, will likely achieve the objectives.

\section{CONCLUSION}

Specialized metabolites produced by plants, especially medicinal plants, have been important sources of drugs, drug precursors, and dietary supplements. While plants use these metabolites to protect themselves from biotic and abiotic stresses, the natural toxicity of specialized metabolites can inhibit growth and development. The production of specialized metabolites can be increased to some extent by modifying the pathway enzymes or regulatory proteins associated with their biosynthesis in plants. However, systematically reprogramming specialized metabolism signaling pathways in plants can be an equally effective or even a better strategy.

The kinase-initiated cascades responsible for signal perception and transduction seem to share many similarities, although the specialized metabolites produced by different plants vary significantly. For example, Arabidopsis, $S$. miltiorrhiza, tobacco, and C. roseus recruit the MAPK cascade to activate the biosynthesis of structurally diverse camalexin, tanshinone, and MIAs (Xie et al. 2020; Raina et al. 2012; Xu et al. 2008; De Boer et al. 2011; Paul et al. 2017). Therefore, creating transgenic lines targeting PKs may lead to altered metabolic outcomes in many medicinal plant species. Based on the assumption that kinase functions are conserved across the plant kingdom, it is possible to engineer the endangered medicinal plants which lack sufficient genomic information. However, as stable transformation protocol is not available for many medicinal plants, transient-based reprogramming (cite the new mol plant paper), hairy roots, cell cultures, or modified Agrobacterium-mediated gene delivery and 
virus-based gene silencing are effective alternatives (Ma et al. 2020; Maher et al. 2020). A recent study demonstrates a versatile and efficient Agrobacterium and RNA viral replicon-based transient expression system that can be used to reprogram developmental and hormone signaling pathways in a wide range of plant species (Torti et al. 2021). Such an approach can potentially be used for PKs to reprogram specialized metabolism in plants.

Other PKs, such as NAD kinase (NADK) and targets of rapamycin kinase (TOR), play critical roles in regulating plant development. However, the roles of these kinases in regulating plant specialized metabolism have not been well studied. NADK is the only known enzyme that phosphorylates $\mathrm{NAD}(\mathrm{H})$ (reviewed in Waller et al. 2009). NADK regulates NADP(H)-dependent metabolism, including the xanthophyll cycle and fatty acid $\beta$ oxidation in plants (Waller et al. 2009). Furthermore, the emerging studies suggest a high potential for plant improvement by targeting TORs (reviewed in Xiong and Sheen 2014; Ryabova et al. 2019). Deciphering the functions of NADK and TOR in plant specialized metabolism requires more investigations.

PKs are key regulators of plant growth and defense. Metabolic reprogramming of medicinal plants is of great value for the modern society. Creating designer plants with higher yield of specialized metabolites but minor growth penalty by targeting PKs is a proven alternative to those achieved by targeting TFs and enzymes.

Acknowledgements This work is supported partially by the Harold R. Burton Endowed Professorship to L.Y. and by the grant 2018530000241001 from the Yunnan Tobacco Company.

\section{Declarations}

Conflict of interest All the authors declare no conflict of interest.

\section{References}

Hussein AR, El-Anssary AA (2019) Plants secondary metabolites: the key drivers of the pharmacological actions of medicinal plants. In: Builders PF (ed) Herbal medicine. IntechOpen, pp 12-30

Anand U, Jacobo-Herrera N, Altemimi A, Lakhssassi N (2019) A comprehensive review on medicinal plants as antimicrobial therapeutics: potential avenues of biocompatible drug discovery. Metabolites 9(11):258-288

Asai T, Tena G, Plotnikova J, Willmann MR, Chiu W-L, GomezGomez L, Boller T, Ausubel FM, Sheen J (2002) MAP kinase signalling cascade in Arabidopsis innate immunity. Nature 415(6875): 977-983

Ashraf MA, Iqbal M, Rasheed R, Hussain I, Riaz M, Arif MS (2018) Chapter 8-environmental stress and secondary metabolites in plants: an overview. In: Ahmad P, Ahanger MA, Singh VP,
Tripathi DK, Alam P, Alyemeni MN (eds) Plant metabolites and regulation under environmental stress. Academic Press, pp 153-167

Bennett RN, Wallsgrove RM (1994) Secondary metabolites in plant defence mechanisms. New Phytol 127(4):617-633

Bigeard J, Hirt H (2018) Nuclear Signaling of plant MAPKs. Front Plant Sci 9:469-469

Birkenbihl RP, Diezel C, Somssich IE (2012) Arabidopsis WRKY33 is a key transcriptional regulator of hormonal and metabolic responses toward botrytis cinerea infection. Plant Physiol 159(1):266-285

Boller T, Felix G (2009) A renaissance of elicitors: perception of microbe-associated molecular patterns and danger signals by pattern-recognition receptors. Annu Rev Plant Biol 60(1):379-406

Borevitz JO, Xia Y, Blount J, Dixon RA, Lamb C (2000) Activation tagging identifies a conserved MYB regulator of phenylpropanoid biosynthesis. Plant Cell 12(12):2383-2394

Borghi L (2010) Inducible gene expression systems for plants. Met Mol Biol 655:65-75

Bourgaud F, Gravot A, Milesi S, Gontier E (2001) Production of plant secondary metabolites: a historical perspective. Plant Sci 161(5):839-851

Campos-Martin JM, Blanco-Brieva G, Fierro JL (2006) Hydrogen peroxide synthesis: an outlook beyond the anthraquinone process. Angew Chem Int Ed Engl 45(42):6962-6984

Cao Y, Li K, Li Y, Zhao X, Wang L (2020) MYB transcription factors as regulators of secondary metabolism in plants. Biology $9(3): 61-77$

Chapman JM, Muhlemann JK, Gayomba SR, Muday GK (2019) RBOH-dependent ROS synthesis and ROS scavenging by plant specialized metabolites to modulate plant development and stress responses. Chem Res Toxicol 32(3):370-396

Chong J, Poutaraud A, Hugueney P (2009) Metabolism and roles of stilbenes in plants. Plant Sci 177(3):143-155

Christie PJ, Alfenito MR, Walbot V (1994) Impact of lowtemperature stress on general phenylpropanoid and anthocyanin pathways: enhancement of transcript abundance and anthocyanin pigmentation in maize seedlings. Planta 194(4):541-549

Cominelli E, Gusmaroli G, Allegra D, Galbiati M, Wade HK, Jenkins GI, Tonelli C (2008) Expression analysis of anthocyanin regulatory genes in response to different light qualities in Arabidopsis thaliana. J Plant Physiol 165(8):886-894

Coppola M, Diretto G, Digilio MC, Woo SL, Giuliano G, Molisso D, Pennacchio F, Lorito M, Rao R (2019) Transcriptome and metabolome reprogramming in tomato plants by trichoderma harzianum strain T22 primes and enhances defense responses against aphids. Front Physiol 10:745-766

Couto D, Zipfel C (2016) Regulation of pattern recognition receptor signalling in plants. Nature Rev Immunology 16(9):537-552

Cui F, Sun W, Kong X (2018) RLCKs bridge plant immune receptors and MAPK cascades. Trends Plant Sci 23(12):1039-1041

D'Auria JC, Gershenzon J (2005) The secondary metabolism of Arabidopsis thaliana: growing like a weed. Curr Opin Plant Biol 8(3):308-316

Dai N, Schaffer A, Petreikov M, Shahak Y, Giller Y, Ratner K, Levine A, Granot D (1999) Overexpression of Arabidopsis hexokinase in tomato plants inhibits growth, reduces photosynthesis, and induces rapid senescence. Plant Cell 11(7):1253-1266

De Boer K, Tilleman S, Pauwels L, Vanden Bossche R, De Sutter V, Vanderhaeghen R, Hilson P, Hamill JD, Goossens A (2011) APETALA2/ETHYLENE RESPONSE FACTOR and basic helixloop-helix tobacco transcription factors cooperatively 
mediate jasmonate-elicited nicotine biosynthesis. Plant J 66(6):1053-1065

Der Fits V, Memelink LJ (2000) ORCA3, a jasmonate-responsive transcriptional regulator of plant primary and secondary metabolism. Science 289(5477):295-297

Desaki Y, Miyata K, Suzuki M, Shibuya N, Kaku H (2018) Plant immunity and symbiosis signaling mediated by LysM receptors. Innate Immun 24(2):92-100

Detarsio E, Wheeler MCG, Campos Bermúdez VA, Andreo CS, MaF D (2003) Maize C4 NADP-malic enzyme: expression in escherichia coli and characterization of site-directed mutants at the putative nucleotide-binding sites*. J Biol Chem 278(16):13757-13764

Diener AC, Ausubel FM (2005) RESISTANCE TO FUSARIUM OXYSPORUM 1, a dominant arabidopsis disease-resistance gene Is Not Race Specific. Genetics 171(1):305-321

Ding K, Pei T, Bai Z, Jia Y, Ma P, Liang Z (2017) SmMYB36, a novel R2R3-MYB transcription factor, enhances tanshinone accumulation and decreases phenolic acid content in salvia miltiorrhiza hairy roots. Sci Rep 7(1):1-15

Dixon RA (2001) Natural products and plant disease resistance. Nature 411(6839):843-847

Dugé de Bernonville T, Carqueijeiro I, Lanoue A, Lafontaine F, Sánchez Bel P, Liesecke F, Musset K, Oudin A, Glévarec G, Pichon O, Besseau S, Clastre M, St-Pierre B, Flors V, Maury S, Huguet E, O'Connor SE, Courdavault V (2017) Folivory elicits a strong defense reaction in Catharanthus roseus: metabolomic and transcriptomic analyses reveal distinct local and systemic responses. Sci Rep 7(1):40453

Ebel J (1979) Elicitor-induced phytoalexin synthesis in soybean (glycine max). In: Luckner M, Schreiber K (eds) Regulation of secondary product and plant hormone metabolism. Pergamon, pp 155-162

Erb M, Reymond P (2019) Molecular interactions between plants and insect herbivores. Annu Rev Plant Biol 70(1):527-557

Frerigmann H, Glawischnig E, Gigolashvili T (2015) The role of MYB34, MYB51 and MYB122 in the regulation of camalexin biosynthesis in Arabidopsis thaliana. Front Plant Sci 6:654-665

Glawischnig E (2007) Camalexin. Phytochem 68(4):401-406

Gomi K (2020) Jasmonic Acid: An Essential Plant Hormone. Int J Mol Sci 21:1261

Gonzalez A, Zhao M, Leavitt JM, Lloyd AM (2008) Regulation of the anthocyanin biosynthetic pathway by the TTG1/bHLH/Myb transcriptional complex in Arabidopsis seedlings. Plant J 53(5):814-827

Gorlenko CL, Kiselev HY, Budanova EV, Zamyatnin AA Jr, Ikryannikova LN (2020) Plant secondary metabolites in the battle of drugs and drug-resistant bacteria: new heroes or worse clones of antibiotics? Antibiotics (basel) 9(4):170-191

Group M, Ichimura K, Shinozaki K, Tena G, Sheen J, Henry Y, Champion A, Kreis M, Zhang S, Hirt H, Wilson C, Heberle-Bors E, Ellis BE, Morris PC, Innes RW, Ecker JR, Scheel D, Klessig DF, Machida Y, Mundy J, Ohashi Y, Walker JC (2002) Mitogenactivated protein kinase cascades in plants: a new nomenclature. Trends Plant Sci 7(7):301-308

Halford NG, Hey SJ (2009) Snf1-related protein kinases (SnRKs) act within an intricate network that links metabolic and stress signalling in plants. Biochem J 419(2):247-259

Harkenrider M, Sharma R, De Vleesschauwer D, Tsao L, Zhang X, Chern M, Canlas P, Zuo S, Ronald PC (2016) Overexpression of rice wall-associated kinase 25 (OsWAK25) alters resistance to bacterial and fungal pathogens. PLOS ONE 11(1):1-16

Hartmann T (2008) The lost origin of chemical ecology in the late 19th century. Proc Natl Acad Sci USA 105(12):4541-4546
He X, Wang C, Wang H, Li L, Wang C (2020) The Function of MAPK CASCADES IN RESPONSE TO VARIOUS STRESSES IN HORTICULTURAL PLANTS. Front Plant Sci 11:952-964

Hu D-G, Sun C-H, Zhang Q-Y, An J-P, You C-X, Hao Y-J (2016a) Glucose sensor MdHXK1 phosphorylates and stabilizes MdbHLH3 to promote anthocyanin biosynthesis in apple. PLOS Genet 12(8):1-27

Hu DG, Ma QJ, Sun CH, Sun MH, You CX, Hao YJ (2016b) Overexpression of MdSOS2L1, a CIPK protein kinase, increases the antioxidant metabolites to enhance salt tolerance in apple and tomato. Physiol Plant 156(2):201-214

Hu K, Cao J, Zhang J, Xia F, Ke Y, Zhang H, Xie W, Liu H, Cui Y, Cao Y, Sun X, Xiao J, Li X, Zhang Q, Wang S (2017) Improvement of multiple agronomic traits by a disease resistance gene via cell wall reinforcement. Nat Plants 3:1-9

Hua WP, Zhang Y, Song J, Zhao LJ, Wang ZZ (2011) De novo transcriptome sequencing in Salvia miltiorrhiza to identify genes involved in the biosynthesis of active ingredients. Genomics 98(4):272-279

Isah $\mathrm{T}$ (2019) Stress and defense responses in plant secondary metabolites production. Biol Res 52(39):1-25

Jia Y, Bai Z, Pei T, Ding K, Liang Z, Gong Y (2017) The protein kinase SmSnRK2.6 positively regulates phenolic acid biosynthesis in salvia miltiorrhiza by interacting with SmAREB1. Front Plant Sci 8(1384):1-16

Jiang M, Chu Z (2018) Comparative analysis of plant MKK gene family reveals novel expansion mechanism of the members and sheds new light on functional conservation. BMC Genom 19(1):407

Jones ME (1953) Albrecht kossel, a biographical sketch. Yale J Biol Med 26(1):80-97

Jones JDG, Dangl JL (2006) The plant immune system. Nature 444(7117):323-329

Kadota Y, Sklenar J, Derbyshire P, Stransfeld L, Asai S, Ntoukakis V, Jones JD, Shirasu K, Menke F, Jones A, Zipfel C (2014) Direct regulation of the NADPH oxidase RBOHD by the PRRassociated kinase BIK1 during plant immunity. Mol Cell 54(1):43-55

Kelly G, David-Schwartz R, Sade N, Moshelion M, Levi A, Alchanatis V, Granot D (2012) The pitfalls of transgenic selection and new roles of AtHXK1: a high level of AtHXK1 expression uncouples hexokinase1-dependent sugar signaling from exogenous sugar. Plant Physiol 159(1):47-51

Kishi-Kaboshi M, Takahashi A, Hirochika H (2010) MAMPresponsive MAPK cascades regulate phytoalexin biosynthesis. Plant Signal Behav 5(12):1653-1656

Kutchan TM (2001) Ecological arsenal and developmental dispatcher. The paradigm of secondary metabolism. Plant Physiol 125(1):58-60

Lee MJ, Yaffe MB (2016) Protein regulation in signal transduction. Cold Spring Harb Perspect Biol 8(6):a005918

Lee J, Eschen-Lippold L, Lassowskat I, Böttcher C, Scheel D (2015) Cellular reprogramming through mitogen-activated protein kinases. Front Plant Sci 6:940-940

Lehti-Shiu MD, Shiu S-H (2012) Diversity, classification and function of the plant protein kinase superfamily. Philos Trans R Soc Lond B Biol Sci 367(1602):2619-2639

Lenka SK, Nims NE, Vongpaseuth K, Boshar RA, Roberts SC, Walker EL (2015) Jasmonate-responsive expression of paclitaxel biosynthesis genes in Taxus cuspidata cultured cells is negatively regulated by the bHLH transcription factors TcJAMYC1, TcJAMYC2, and TcJAMYC4. Front Plant Sci 6(115):78-101

Li CY, Leopold AL, Sander GW, Shanks JV, Zhao L, Gibson SI (2013) The ORCA2 transcription factor plays a key role in regulation 
of the terpenoid indole alkaloid pathway. BMC Plant Biol 13(1):155-172

Li L, Li M, Yu L, Zhou Z, Liang X, Liu Z, Cai G, Gao L, Zhang X, Wang Y, Chen S, Zhou J-M (2014a) The FLS2-associated kinase BIK1 directly phosphorylates the NADPH oxidase RbohD to control plant immunity. Cell Host Microbe 15(3):329-338

Li W, Liu W, Wei H, He Q Chen J, Zhang B, Zhu S (2014b) Speciesspecific expansion and molecular evolution of the 3-hydroxy3-methylglutaryl coenzyme a reductase (HMGR) gene family in plants. PLOS ONE 9(4):e94172

Li S, Wang W, Gao J, Yin K, Wang R, Wang C, Petersen M, Mundy J, Qiu J-L (2016) MYB75 phosphorylation by MPK4 Is required for light-induced anthocyanin accumulation in Arabidopsis. Plant Cell 28(11):2866-2883

Li B, Fan R, Guo S, Wang P, Zhu X, Fan Y, Chen Y, He K, Kumar A, Shi J, Wang Y, Li L, Hu Z, Song C-P (2019) The Arabidopsis MYB transcription factor, MYB111 modulates salt responses by regulating flavonoid biosynthesis. Environ Exp Bot 166:103807-103816

Liang X, Zhou JM (2018) Receptor-like cytoplasmic kinases: central players in plant receptor kinase-mediated signaling. Annu Rev Plant Biol 69:267-299

Liu Y, Patra B, Pattanaik S, Wang Y, Yuan L (2019) GATA and phytochrome interacting factor transcription factors regulate light-induced vindoline biosynthesis in Catharanthus roseus. Plant Physiol 180(3):1336-1350

Liu X, Singh SK, Patra B, Liu Y, Wang B, Wang J, Pattanaik S, Yuan L (2021) Protein-phosphatase NtPP2C2b and MAP-kinase NtMPK4 act in concert to modulate nicotine biosynthesis. J Exp Bot 72:1661-1676

Lu D, Wu S, Gao X, Zhang Y, Shan L, He P (2010) A receptor-like cytoplasmic kinase, BIK1, associates with a flagellin receptor complex to initiate plant innate immunity. Proc Natl Acad Sci 107(1):496-501

Luo J, Wang X, Feng L, Li Y, He J-X (2017) The mitogen-activated protein kinase kinase 9 (MKK9) modulates nitrogen acquisition and anthocyanin accumulation under nitrogen-limiting condition in Arabidopsis. Biochem Biophys Res Commun 487(3):539-544

Ma X, Zhang X, Liu H, Li Z (2020) Highly efficient DNA-free plant genome editing using virally delivered CRISPR-Cas9. Nature Plants 6(7):773-779

Maher MF, Nasti RA, Vollbrecht M, Starker CG, Clark MD, Voytas DF (2020) Plant gene editing through de novo induction of meristems. Nature Biotech 38(1):84-89

Mala D, Awasthi S, Sharma NK, Swarnkar MK, Shankar R, Kumar S (2021) Comparative transcriptome analysis of Rheum australe, an endangered medicinal herb, growing in its natural habitat and those grown in controlled growth chambers. Sci Rep 11(1):3702-3718

Mao G, Meng X, Liu Y, Zheng Z, Chen Z, Zhang S (2011) Phosphorylation of a WRKY transcription factor by two pathogen-responsive MAPKs drives phytoalexin biosynthesis in Arabidopsis. Plant Cell 23(4):1639-1653

Menke FL, Champion A, Kijne JW, Memelink J (1999) A novel jasmonate- and elicitor-responsive element in the periwinkle secondary metabolite biosynthetic gene Str interacts with a jasmonate- and elicitor-inducible AP2-domain transcription factor, ORCA2. Embo J 18(16):4455-4463

Menu T, Saglio P, Granot D, Dai N, Raymond P, Ricard B (2004) High hexokinase activity in tomato fruit perturbs carbon and energy metabolism and reduces fruit and seed size. Plant Cell Environ 27(1):89-98

Meraj TA, Fu J, Raza MA, Zhu C, Shen Q Xu D, Wang Q (2020) Transcriptional factors regulate plant stress responses through mediating secondary metabolism. Genes 11(4):346-364

Mogensen TH (2009) Pathogen recognition and inflammatory signaling in innate immune defenses. Clin Microbiol Rev 22(2):240-273

Nakabayashi R, Yonekura-Sakakibara K, Urano K, Suzuki M, Yamada Y, Nishizawa T, Matsuda F, Kojima M, Sakakibara H, Shinozaki K, Michael AJ, Tohge T, Yamazaki M, Saito K (2014) Enhancement of oxidative and drought tolerance in Arabidopsis by overaccumulation of antioxidant flavonoids. Plant J 77(3):367-379

Nakagami H, Pitzschke A, Hirt H (2005) Emerging MAP kinase pathways in plant stress signalling. Trends Plant Sci 10(7):339-346

Neuss R, Neuss M N (1990) Therapeutic use of bisindole alkaloids from Catharanthus. In: Brossi A, Suffness M (eds) The alkaloids: antitumor bisindole alkaloids from Catharanthus roseus. Academic Press, 37:229-240

Ng DWK, Abeysinghe JK, Kamali M (2018) Regulating the regulators: the control of transcription factors in plant defense signaling. Int J Mol Sci 19(12):3737-3755

Noman A, Aqeel M, Lou Y (2019) PRRs and NB-LRRs: from signal perception to activation of plant innate immunity. Int J Mol Sci 20(8):1882-1894

Pan Y-j, Lin Y-c, Yu B-f, Zu Y-g, Yu F, Tang Z-H (2018) Transcriptomics comparison reveals the diversity of ethylene and methyl-jasmonate in roles of TIA metabolism in Catharanthus roseus. BMC Genomics 19(1):508-522

Park J-Y, Kim JH, Kim YM, Jeong HJ, Kim DW, Park KH, Kwon H-J, Park S-J, Lee WS, Ryu YB (2012) Tanshinones as selective and slow-binding inhibitors for SARS-CoV cysteine proteases. Bioorg Med Chem 20(19):5928-5935

Patra B, Schluttenhofer C, Wu Y, Pattanaik S, Yuan L (2013) Transcriptional regulation of secondary metabolite biosynthesis in plants. Biochim Biophys Acta 11:1236-1247

Patra B, Pattanaik S, Schluttenhofer C, Yuan L (2018) A network of jasmonate-responsive bHLH factors modulate monoterpenoid indole alkaloid biosynthesis in Catharanthus roseus. New Phytol 217(4):1566-1581

Paul P, Singh SK, Patra B, Sui X, Pattanaik S, Yuan L (2017) A differentially regulated AP2/ERF transcription factor gene cluster acts downstream of a MAP kinase cascade to modulate terpenoid indole alkaloid biosynthesis in Catharanthus roseus. New Phytol 213(3):1107-1123

Paul P, Singh SK, Patra B, Liu X, Pattanaik S, Yuan L (2020) Mutually regulated AP2/ERF gene clusters modulate biosynthesis of specialized metabolites in plants. Plant Physiol 182(2):840-857

Pichersky E, Lewinsohn E (2011) Convergent evolution in plant specialized metabolism. Annu Rev Plant Biol 62(1):549-566

Pietra F (1997) Secondary metabolites from marine microorganisms: bacteria, protozoa, algae and fungi. Achievements and prospects. Nat Prod Rep 14(5):453-464

Pietta PG (2000) Flavonoids as antioxidants. J Nat Prod 63(7):1035-1042

Pott DM, Osorio S, Vallarino JG (2019) From central to specialized metabolism: an overview of some secondary compounds derived from the primary metabolism for their role in conferring nutritional and organoleptic characteristics to fruit. Front Plant Sci 10:1-19

Prasanna V, Prabha TN, Tharanathan RN (2007) Fruit ripening phenomena-an overview. Crit Rev Food Sci Nutr 47(1):1-19

Qiu JL, Fiil BK, Petersen K, Nielsen HB, Botanga CJ, Thorgrimsen S, Palma K, Suarez-Rodriguez MC, Sandbech-Clausen S, Lichota J, Brodersen P, Grasser KD, Mattsson O, Glazebrook J, Mundy J, Petersen M (2008) Arabidopsis MAP kinase 4 regulates gene 
expression through transcription factor release in the nucleus. EMBO J 27(16):2214-2221

Qu Y, Safonova O, De Luca V (2019) Completion of the canonical pathway for assembly of anticancer drugs vincristine/vinblastine in Catharanthus roseus. Plant J 97:257-266

Raina S, Wankhede D, Jaggi M, Singh P, Jalmi S, Raghuram B, Sheikh A, Sinha A (2012) CrMPK3, a mitogen activated protein kinase from Catharanthus roseus and its possible role in stress induced biosynthesis of monoterpenoid indole alkaloids. BMC Plant Biol 12(1):134-255

Ramakrishna A, Ravishankar GA (2011) Influence of abiotic stress signals on secondary metabolites in plants. Plant Signal Behav 6(11):1720-1731

Ren D, Liu Y, Yang K-Y, Han L, Mao G, Glazebrook J, Zhang S (2008) A fungal-responsive MAPK cascade regulates phytoalexin biosynthesis in Arabidopsis. Proc Natl Acad Sci USA 105(14):5638-5643

Rhoades DF (1977) Integrated antiherbivore, antidesiccant and ultraviolet screening properties of creosotebush resin. Biochem Syst Ecol 5(4):281-290

Robertlee J, Kobayashi K, Suzuki M, Muranaka T (2017) AKIN10, a representativeArabidopsis SNF1-related protein kinase 1 (SnRK1), phosphorylates and downregulates plant HMGCoA reductase. FEBS Lett 591(8):1159-1166

Rodriguez-Concepcion M, Boronat A (2002) Elucidation of the methylerythritol phosphate pathway for isoprenoid biosynthesis in bacteria and plastids. A metabolic milestone achieved through genomics. Plant Physiol 130(3):1079-1089

Ryabova LA, Robaglia C, Meyer C (2019) Target of Rapamycin kinase: central regulatory hub for plant growth and metabolism. J Exp Bot 70(8):2211-2216

Seybold H, Trempel F, Ranf S, Scheel D, Romeis T, Lee J (2014) $\mathrm{Ca}^{2+}$ signalling in plant immune response: from pattern recognition receptors to $\mathrm{Ca}^{2+}$ decoding mechanisms. New Phytol 204(4):782-790

Shoji T, Yuan L (2021) ERF gene clusters: working together to regulate metabolism. Trends Plant Sci 26(1):23-32

Singh SK, Patra B, Paul P, Liu Y, Pattanaik S, Yuan L (2020) Revisiting the ORCA gene cluster that regulates terpenoid indole alkaloid biosynthesis in Catharanthus roseus. Plant Sci 293:110408-111021

Singh SK, Patra B, Paul P, Liu Y, Pattanaik S, Yuan L (2021) BHLH IRIDOID SYNTHESIS 3 is a member of a bHLH gene cluster regulating terpenoid indole alkaloid biosynthesis in Catharanthus roseus. Plant Direct 5(1):1-13

Suttipanta N, Pattanaik S, Kulshrestha M, Patra B, Singh SK, Yuan L (2011) The transcription factor CrWRKY1 positively regulates the terpenoid indole alkaloid biosynthesis in Catharanthus roseus. Plant Physiol 157(4):2081-2093

Tahmasebi A, Ebrahimie E, Pakniyat H, Ebrahimi M, MohammadiDehcheshmeh M (2019) Insights from the Echinacea purpurea (L.) moench transcriptome: global reprogramming of gene expression patterns towards activation of secondary metabolism pathways. Ind Crops Prod 132:365-376

Taj G, Agarwal P, Grant M, Kumar A (2010) MAPK machinery in plants: recognition and response to different stresses through multiple signal transduction pathways. Plant Signal Behav 5(11):1370-1378

Torti S, Schlesier R, Thümmler A, Bartels D, Römer P, Koch B, Werner S, Panwar V, Kanyuka K, Nv W, Jones JDG, Hause G, Giritch A, Gleba Y (2021) Transient reprogramming of crop plants for agronomic performance. Nature Plants 7(2):159-171

Turner WB (1971) Fungal metabolites. Academic Press, London

Van Moerkercke A, Steensma P, Schweizer F, Pollier J, Gariboldi I, Payne R, Vanden Bossche R, Miettinen K, Espoz J, Purnama
PC, Kellner F, Seppänen-Laakso T, O'Connor SE, Rischer H, Memelink J, Goossens A (2015) The bHLH transcription factor BIS1 controls the iridoid branch of the monoterpenoid indole alkaloid pathway in Catharanthus roseus. Proc Natl Acad Sci USA 112(26):8130-8135

Van Moerkercke A, Steensma P, Gariboldi I, Espoz J, Purnama PC, Schweizer F, Miettinen K, Vanden Bossche R, De Clercq R, Memelink J, Goossens A (2016) The basic helix-loop-helix transcription factor BIS2 is essential for monoterpenoid indole alkaloid production in the medicinal plant Catharanthus roseus. Plant J 88(1):3-12

Verma M, Ghangal R, Sharma R, Sinha AK, Jain M (2014) Transcriptome analysis of catharanthus roseus for gene discovery and expression profiling. PLOS ONE 9(7):1-11

Vom Endt D, Kijne JW, Memelink J (2002) Transcription factors controlling plant secondary metabolism: what regulates the regulators? Phytochem 61(2):107-114

Vrebalov J, Ruezinsky D, Padmanabhan V, White R, Medrano D, Drake R, Schuch W, Giovannoni J (2002) A MADS-box gene necessary for fruit ripening at the tomato ripening-inhibitor locus. Science 296(5566):343-346

Waller JC, Dhanoa PK, Schumann U, Mullen RT, Snedden WA (2009) Subcellular and tissue localization of NAD kinases from Arabidopsis: compartmentalization of de novo NADP biosynthesis. Planta 231(2):305-317

Wang J, Chai J (2020) Structural insights into the plant immune receptors PRRs and NLRs. Plant Physiol 182(4):1566-1581

Wang Y-C, Wang Y-R (2011) Levels of phenolic acids and tanshinones in salvia miltiorrhiza bge. cv. sativa and salvia bowleyanae dunn roots during growth. J Biol Active Prod Nature 1(1):7-18

Wang H, Chevalier D, Larue C, Ki Cho S, Walker JC (2007) The protein phosphatases and protein kinases of Arabidopsis thaliana. Arabidopsis Book 5:e0106-e0106

Wang X, Peng F, Li M, Yang L, Li G (2012) Expression of a heterologous SnRK1 in tomato increases carbon assimilation, nitrogen uptake and modifies fruit development. J Plant Physiol 169(12):1173-1182

Wang S, Alseekh S, Fernie AR, Luo J (2019) The structure and function of major plant metabolite modifications. Mol Plant 12(7):899-919

Waszczak C, Carmody M, Kangasjärvi J (2018) Reactive oxygen species in plant signaling. Annu Rev Plant Biol 69(1):209-236

Weathers PJ, Elkholy S, Wobbe KK (2006) Artemisinin: the biosynthetic pathway and its regulation in Artemisia annua, a terpenoid-rich species. In Vitro Cell Dev Biol Plant 42(4):309-317

Wersch RV, Gao F, Zhang Y (2018) Mitogen-activated protein kinase kinase 6 negatively regulates anthocyanin induction in Arabidopsis. Plant Signal Behav 13(10):e1526001-e1526003

Wurtzel ET, Kutchan TM (2016) Plant metabolism, the diverse chemistry set of the future. Science 353(6305):1232-1236

Xie Y, Ding M, Zhang B, Yang J, Pei T, Ma P, Dong J (2020) Genomewide characterization and expression profiling of MAPK cascade genes in Salvia miltiorrhiza reveals the function of SmMAPK3 and SmMAPK1 in secondary metabolism. BMC Genom 21(1):2-27

Xiong Y, Sheen J (2014) The role of target of rapamycin signaling networks in plant growth and metabolism. Plant Physiol 164(2):499-523

Xu D, Nussinov R (1998) Favorable domain size in proteins. Fold Des 3(1):11-17

Xu J, Li Y, Wang Y, Liu H, Lei L, Yang H, Liu G, Ren D (2008) Activation of MAPK kinase 9 induces ethylene and camalexin 
biosynthesis and enhances sensitivity to salt stress in Arabidopsis. J Biol Chem 283(40):26996-27006

Xu J, Meng J, Meng X, Zhao Y, Liu J, Sun T, Liu Y, Wang Q, Zhang S (2016) Pathogen-responsive MPK3 and MPK6 reprogram the biosynthesis of indole glucosinolates and their derivatives in Arabidopsis immunity. Plant Cell 28(5):1144-1167

Yang C-Q Fang X, Wu X-M, Mao Y-B, Wang L-J, Chen X-Y (2012) Transcriptional regulation of plant secondary metabolism. J Integr Plant Biol 54(10):703-712

Yang P, Praz C, Li B, Singla J, Robert CAM, Kessel B, Scheuermann D, Lüthi L, Ouzunova M, Erb M, Krattinger SG, Keller B (2019) Fungal resistance mediated by maize wall-associated kinase ZmWAK-RLK1 correlates with reduced benzoxazinoid content. New Phytol 221(2):976-987

Yang L, Zhang Y, Guan R, Li S, Xu X, Zhang S, Xu J (2020) Coregulation of indole glucosinolates and camalexin biosynthesis by CPK5/CPK6 and MPK3/MPK6 signaling pathways. J Integr Plant Biol 62(11):1780-1796

Yazaki K, Arimura G-i, Ohnishi T (2017) 'Hidden' terpenoids in plants: their biosynthesis, localization and ecological roles. Plant Cell Physiol 58(10):1615-1621

Yeo Y-S, Nybo SE, Chittiboyina AG, Weerasooriya AD, Wang Y-H, Góngora-Castillo E, Vaillancourt B, Buell CR, Dellapenna D, Celiz MD, Jones AD, Wurtele ES, Ransom N, Dudareva N, Shaaban KA, Tibrewal N, Chandra S, Smillie T, Khan IA, Coates RM, Watt DS, Chappell J (2013) Functional identification of valerena-1,10-diene synthase, a terpene synthase catalyzing a unique chemical cascade in the biosynthesis of biologically active sesquiterpenes in valeriana officinalis. J Biol Chem 288(5):3163-3173

Yu W, Peng F, Xiao Y, Wang G, Luo J (2018) Overexpression of PpSnRK1 $\alpha$ in tomato promotes fruit ripening by enhancing ripening inhibitor regulation pathway. Front Plant Sci 9:1-10
Zhang H, Hedhili S, Montiel G, Zhang Y, Chatel G, Pré M, Gantet P, Memelink J (2011) The basic helix-loop-helix transcription factor CrMYC2 controls the jasmonate-responsive expression of the ORCA genes that regulate alkaloid biosynthesis in Catharanthus roseus. Plant J 67(1):61-71

Zhang F, Fu X, Lv Z, Lu X, Shen Q, Zhang L, Zhu M, Wang G, Sun X, Liao Z, Tang K (2015) A basic leucine zipper transcription factor, AabZIP1, connects abscisic acid signaling with artemisinin biosynthesis in Artemisia annua. Mol Plant 8(1):163-175

Zhang N, Zhang B, Zuo W, Xing Y, Konlasuk S, Tan G, Zhang Q, Ye J, Xu M (2017) Cytological and molecular characterization of ZmWAK-mediated head-smut resistance in maize. Mol Plant Microbe Interact 30(6):455-465

Zhang F, Xiang L, Yu Q, Zhang H, Zhang T, Zeng J, Geng C, Li L, Fu X, Shen Q, Yang C, Lan X, Chen M, Tang K, Liao Z (2018) Artemisinin biosynthesis promoting kinase 1 positively regulates artemisinin biosynthesis through phosphorylating AabZIP1. J Exp Bot 69(5):1109-1123

Zhou N, Tootle TL, Glazebrook J (1999) Arabidopsis PAD3, a gene required for camalexin biosynthesis, encodes a putative cytochrome P450 monooxygenase. Plant Cell 11(12):2419-2428

Zhou J, Wang X, He Y, Sang T, Wang P, Dai S, Zhang S, Meng X (2020) Differential phosphorylation of the transcription factor WRKY33 by the protein kinases CPK5/CPK6 and MPK3/MPK6 cooperatively regulates camalexin biosynthesis in Arabidopsis. Plant Cell 32(8):2621-2640

Zipfel C (2014) Plant pattern-recognition receptors. Trends Immunol 35(7):345-351 